\title{
Emerging trends of nosocomial Citrobacter species surgical wound infection: concern for infection control
}

\author{
Thapa $\mathrm{B}^{1}$, Karn $\mathrm{D}^{2}$, Mahat $\mathrm{K}^{3}$
}

${ }^{1}$ Lecturer, Department of Microbiology, Kathmandu medical College, Kathmandu,

${ }^{2}$ Lecturer, Department of Dermatology, Kathmandu University School of Medical Science, Dhulikhel,

${ }^{3}$ Mahidol Univeristy Alumni Association Nepal, Kathmandu

\section{Correspondence}

Dr. Badri Thapa

Lecturer, Department of Microbiology, Kathmandu Medical College, Nepal

E-mail: badri_bishal@yahoo.com

\begin{abstract}
Background

Surgical wound infection is a common problem among patients who undergo operation. Several factors play important role in this infection process including endemic nosocomial infection without proper infection control measures.
\end{abstract}

\section{Objectives}

To study the occurrence of the pathogens in post-operative wound infections, their antibiotic resistance patterns, and comparison with published reports.

\section{Methods}

Various specimens obtained from the surgical wound during a period of four months were processed for bacteriological culture in the Department of Microbiology, Kathmandu Medical College, Kathmandu. Antibiotic susceptibility test was performed by Kirby-Bauer disk diffusion test for pathogens isolated. The relevant literatures were searched and compared with the present study.

\section{Results}

Among 79 culture positive cultures, Citrobacter sp. (n=23) was most frequently isolated from surgical wound infection. Twenty strains were multidrug resistant. In comparison with other studies, this study highlights the emergence multi-drug resistant Citrobacter $\mathrm{sp}$. as a leading cause of surgical wound infection. E. coli, Staphylococcus aureus, Acinetobacter sp., Klebsiella pneumoniae, Pseudomonas aeruginosa, Proteus sp., and Enterobacter sp. were also isolated from $19,13,10,8,2,2$, and 2 cultures, respectively. More than $50 \%$ of these pathogens were resistant to most of the $\beta$-lactam antibiotics tested and most of them were multi-drug resistant while these pathogens showed variable level of resistance to fluroquinolones and amino glycosides.

\section{Conclusion}

The frequent isolation of multi-drug resistant nosocomial strains of Citrobacter sp. in surgical wound infection is a remarkable trend. This pathogen and their resistant genes could be endemic to the institution and can cause difficult-to-treat infection if infection control committee is not revitalized and infection control strategies are not implemented.

Key words

Citrobacter sp., Multi-drug resistant, surgical wound infection. 


\section{Introduction}

Numerous patients are admitted in a hospital for surgical procedures. The incision site is often complicated by nosocomial wound infection which gives psychological and emotional stress to the patient and to the entire family, significantly delays the hospital discharge, increases the financial burden and raises the morbidity and mortality ${ }^{1}$. The surgical wound infection is common in Nepal and worldwide. The rate of surgical wound infection in Tribhuvan University Teaching Hospital (TUTH), Nepal and the post-operative cesarean wound infection in Patan Hospital was $4 \%$ and $2.76 \%$, respectively ${ }^{2,3}$. Nosocomial wound infection is also a common problem in other hospitals of $\mathrm{Nepal}^{4}$.

Among the pathogens causing nosocomial wound infection coagulase positive Staphylococcus aureus is common and the treatment is often complicated by the isolation of Methicillin-resistant S. aureus (MRSA) and Vancomycin Resistant S. aureus (VRSA) 2,3,5-7. Vancomycin resistance significantly raises the hospital stay to 18.1 days and cost as high as $\$ 27,190^{8}$. Gram negative pathogens like, Escherichia coli, K. pneumoniae, Proteus sp, Enterobacter sp, P. aeruginosa, Acinetobacter baumannii, and Citrobacter sp. also

causes nosocomial wound infection ${ }^{2}$. The commonest Gram negative organisms isolated were P. aeruginosa, E. coli, and K. pneumonia in wound infection ${ }^{2,9}$. The antibiotic resistance in these pathogens is increasing. The emergence of resistance in these pathogens to third generation cephalosporins, carbipenems, tigecycline, colistin and rifampicin has marked the therapeutic deadlock $^{10-12}$. The rates of isolation of multi-drug resistant superbugs are increasing casing havoc in health care settings.

Here, we studied the prevalence of the pathogens in post-operative wound infections, their antibiotic resistance patterns and compared the trends of pathogen occurrence with published reports.

\section{Methods}

This laboratory research was conducted in Department of Microbiology, Kathmandu Medical College in collaboration with Department of Dermatology, Kathmandu University School of Medical Science, Dhulikhel, Nepal.

Bacterial isolates: The bacterial isolates that were isolated in the Department of Microbiology from pus, wound swab, and debrided tissues cultures from postoperative patients admitted in Kathmandu Medical College Teaching Hospital during the period of January to March 2010 were studied. The pathogens were identified using standard phenotypic methods ${ }^{13}$.

Antibiotic susceptibility tests: Disk Diffusion Test was performed to assess the in-vitro activity of different antibiotics to the isolated pathogens. All of the antibiotic disks ( $\mu \mathrm{g} /$ disk) were purchased from HiMedia Laboratories Pvt. Ltd., India except Cefoxitin and Cefazolin which were purchased from Oxoid,UK. The resistant, intermittent, and susceptible zone size was interpreted based on the guidelines of manufacturer which is based on CLSI guidelines. The intermittent zone of resistance was interpreted as resistant. Multi-drug resistant $(\mathrm{MDR})$ isolates were defined as the isolates that were resistant to two or more than two antibiotics tested.

Comparison of pathogens isolated (with published reports): Literature search was performed using the google search engine. The key words used were, surgical wound infection, pathogens, and antibiotic resistance. Three main relevant literatures ${ }^{2,9,14}$ were identified and the data from these studies were compared with the preset study.

\section{Results}

Among 79 pathogens isolated from different specimen sources within four months period, Citrobacter sp. was recovered from 23 cultures (Table 1). Similarly, E. coli, S. aureus, Acinetobacter sp., K. pneumonia, P. aeruginosa, Proteus sp., and Enterobacter sp. were isolated from 19, $13,10,8,2,2$, and 2 cultures, respectively.

More than $50 \%$ of the isolates isolated were resistant to most of the $\beta$-lactam antibiotics tested. Strains of Citobacter sp. $(n=5)$, E. coli $(n=3)$, and Acinetobacter sp. $(n=5)$ were tested for $\beta$-lactam and $\beta$-lactam inhibitor combination (sulphaperazone+sulbactam) and all isolates of Citrobacter sp. and E. coli were susceptible while few strains $(n=2)$ of Acinetobacter sp. were resistant to this combination. Imipenem resistance was noted for one E. coli isolate. Fluroquinolones (Ofloxacin, Ciprofloxacin, and Norfloxacin) were insensitive to most strains of E. coli, Acineotbacter sp., and P. aeruginosa while they were sensitive to other pathogens. Most pathogens were susceptible to the amino glycosides tested.

Among 23 isolates of Citrobacter sp. 20 showed MDR phenotype. Similarly, MDR phenotype of E. coli, S. aureus, Acinetobacter sp., K. pneumonia, P. aeruginosa, Proteus sp., and Enterobacter sp. noted were 17, 6, 9, 6, 2, 2 , and 2 in number, respectively.

Among seven most frequently recovered pathogens from wound infection, S. aureus was the most frequently 
isolated in three published literatures (Table 2). However, this study identified Citrobacter sp. as a commonest pathogen isolated. Citrobacter sp. although less common, they were also isolated among top seven pathogens in tow of those studies. E. coli was the second most common in this study and two other studies. The frequency of distribution of other pathogens varied among different studies.

\section{Discussion}

Nosocomial surgical wound infection in patients attending to hospital is increasing and multidrug resistant pathogens are being isolated. S. aureus, E. coli, are $\mathrm{K}$. pneumoniae were common pathogen to cause wound infection ${ }^{2}$. This study highlighted the emergence of MDR Citrobacter sp. as a leading cause of wound

Table 1: Pathogens isolated from surgical wound infection and their antibiotic resistance patterns

\begin{tabular}{|c|c|c|c|c|c|c|c|c|c|c|c|c|c|c|c|c|c|c|c|c|c|}
\hline \multirow[b]{2}{*}{ Pathogens (n) } & \multicolumn{21}{|c|}{ Resistant isolates (no. of isolates tested) } \\
\hline & $\varangle$ & 蒾 & ¿ & త & ธ & $\underline{\underline{N}}$ & ษ & u & $\mathscr{\bullet}$ & $\because$ & $\varangle$ & $s$ & ơ & ठ & ঊ & 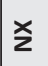 & ৩ & $\stackrel{\check{\alpha}}{\check{\alpha}}$ & ○ & $\vdash$ & - \\
\hline Citrobacter sp. (23) & 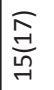 & i & i & $i$ & 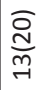 & & 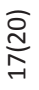 & $\frac{\widehat{n}}{0}$ & $\frac{\bar{J}}{m}$ & $i$ & i & i & i & $\underset{\text { 근 }}{\bar{N}}$ & $\underset{\underset{N}{\mathbb{N}}}{\overline{\mathbb{N}}}$ & $\underset{\underset{\mathrm{J}}{\mathbb{J}}}{\mathbb{J}}$ & 㝘 & $\underset{i n}{\stackrel{\infty}{\leftrightarrows}}$ & 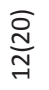 & $i$ & $\bar{\Xi}$ \\
\hline E. coli (19) & 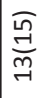 & $\underset{ન}{\vec{F}}$ & $\stackrel{\bar{m}}{\sim}$ & 1 & ન્ & i & 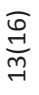 & $\overline{\frac{m}{\sigma}}$ & $\underset{ }{\stackrel{D}{ }}$ & $\frac{\bar{m}}{m}$ & $\frac{\bar{m}}{m}$ & 1 & 1 & $\underset{\infty}{\widehat{\sigma}}$ & $\underset{\sigma}{\stackrel{\infty}{\sigma}}$ & 흠 & 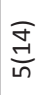 & $\frac{\bar{g}}{\stackrel{m}{m}}$ & $\underset{\sigma}{\stackrel{\vec{J}}{\vec{U}}}$ & 1 & $\underset{ন}{\overparen{I}}$ \\
\hline S. aureus (13) & i & $\underset{\infty}{\mathbb{I}}$ & 1 & $\frac{\sigma}{m}$ & 1 & $\frac{\widehat{n}}{0}$ & 1 & i & i & i & 1 & $\frac{\widehat{D}}{\sigma}$ & $\widehat{\mathbb{a}}$ & $i$ & $\underset{m}{\stackrel{m}{m}}$ & 1 & $\underset{\sim}{\bar{E}}$ & 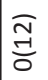 & $\frac{\widehat{Q}}{m}$ & $\underset{m}{\vec{m}}$ & $\frac{\widehat{d}}{0}$ \\
\hline Acinetobacter sp. (10) & $\frac{\bar{\sigma}}{m}$ & i & $\frac{\widehat{J}}{m}$ & & $\frac{\bar{\infty}}{m}$ & $i$ & $\frac{\bar{m}}{m}$ & $\stackrel{\sqrt{n}}{\sim}$ & $\frac{\sqrt{n}}{n n}$ & & i & $i$ & i & $\frac{\bar{T}}{6}$ & 옹 & $\frac{\bar{\theta}}{\omega}$ & $\underset{f}{\partial}$ & 옹 & $\frac{\sqrt{n}}{-1}$ & $\overline{\mathrm{d}}$ & $i$ \\
\hline K. pneumoniae (8) & $\frac{\bar{\infty}}{\sim}$ & 1 & 1 & 1 & $\frac{\bar{E}}{m}$ & 1 & $\frac{\bar{S}}{\mathrm{~N}}$ & 1 & 1 & & 1 & i & 1 & $\underset{-}{\bar{E}}$ & $\stackrel{\bar{\infty}}{\sim}$ & $\frac{\bar{\infty}}{\sim}$ & $\underset{\sim}{\mathbb{E}}$ & $\underset{\sim}{\infty}$ & $\frac{\widehat{\infty}}{f}$ & 1 & 1 \\
\hline P. aeruginosa (2) & $\underset{\sim}{\mathbb{d}}$ & 1 & 1 & i & $\underset{\sim}{\mathbb{N}}$ & 1 & $\underset{\mathrm{d}}{\mathbb{d}}$ & 1 & $\bar{F}$ & $\bar{\Xi}$ & 1 & $i$ & 1 & $\underset{\mathbb{N}}{\bar{d}}$ & $\underset{\mathrm{d}}{\mathrm{d}}$ & $\underset{\sim}{\mathbb{d}}$ & $\underset{ন}{\widehat{D}}$ & $\underset{\sim}{\stackrel{d}{d}}$ & $\underset{\mathbb{N}}{\bar{d}}$ & 1 & 1 \\
\hline Proteus sp. (2) & $\sqrt[\text { a }]{2}$ & 1 & ' & 1 & 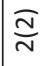 & i & $\frac{\sqrt{n}}{\bar{y}}$ & 1 & 1 & 1 & 1 & 1 & $i$ & $\overline{\frac{2}{0}}$ & $\overline{\frac{1}{0}}$ & $\overline{\mathrm{d}}$ & $\underset{ন}{\stackrel{\Xi}{(}}$ & $\underset{\sim}{\mathbb{d}}$ & $\underset{\exists}{\vec{్}}$ & 1 & 1 \\
\hline Enterobacter sp. (2) & $\underset{\vec{\gamma}}{\widehat{\Xi}}$ & ' & $\underset{\vec{T}}{\vec{I}}$ & $i$ & $\overline{\mathrm{g}}$ & $i$ & $\underset{\exists}{\Xi}$ & i & $\underset{ }{\vec{F}}$ & $\underset{ন}{\vec{F}}$ & $i$ & $i$ & $i$ & $\overline{\frac{D}{\sigma}}$ & $\overline{\mathrm{d}}$ & $\bar{\Xi}$ & $\widehat{\widetilde{d}}$ & $\bar{\Xi}$ & $i$ & $i$ & $i$ \\
\hline
\end{tabular}

"n”-total no. of isolates recovered; “-“, antibiotics not tested; A, Amoxycillin ( $10 \mu \mathrm{g})$; AC, Amoxy-clavulenic acid (20/10 ug); PC, Piperacillin $(100 \mu \mathrm{g})$; CX, Cloxacillin $(30 \mu \mathrm{g})$; CI, Ceftriaxone $(30 \mu \mathrm{g}) ; \mathrm{KZ}$, Cefazolin $(30 \mu \mathrm{g})$; CP, Cephalexin $(30 \mu \mathrm{g})$; SC, Suphoperazone+Salbactam $(75 / 10 \mu \mathrm{g})$; TB, Tobramycin $(10 \mu \mathrm{g}) ; \mathrm{CB}$, Carbenecillin $(100 \mu \mathrm{g})$; CA, Ceftazidime (30 $\mu \mathrm{g})$; VA, Vancomycin (5 $\mu \mathrm{g})$; FOX, Cefoxitin (30 $\mu \mathrm{g})$; OF, Ofloxacin $(5 \mu \mathrm{g})$; CF, Ciprofloaxcin (5 $\mu \mathrm{g})$; NX, Norfloaxin $(10 \mu \mathrm{g})$; G, Gentamicin $(30 \mu \mathrm{g}) ; \mathrm{AK}$, Amikacin ( $10 \mu \mathrm{g})$; CO, Sulphamethoxazole+Trimethoprim $(23.75 / 1.25 \mu \mathrm{g})$; T, Tetracycline $(30 \mu \mathrm{g})$; I, Imipenem $(10 \mu \mathrm{g})$

Table 2: Frequency of pathogens isolated from wound infection in different hospitals and this study

\begin{tabular}{|l|l|l|l|l|}
\hline S.N. & TUTH, 2003 (2) & TUTH, 2003 (14) & MHRC, 2007 (9) & This study \\
\hline 1 & S. aureus & S. aureus & S. aureus & Citrobacter sp. \\
\hline 2 & P. aeruginosa & E. coli & E. coli & E. coli \\
\hline 3 & E. coli & Coagulase negative Staphylococcus sp. & P. aeruginosa & S. aureus \\
\hline 4 & K. pneumoniae & K. pneumoniae & S. pyogenes & Acinetobacter sp. \\
\hline 5 & Acinetobacter sp. & Proteus vulgaris & K. pneumoniae & K. pneumoniae \\
\hline 6 & Citrobacter sp. & Proteus mirabilis & Coagulase negative Staphylococcus sp. & P. aeruginosa \\
\hline 7 & Proteus vulgaris & Citrobacter sp. & -- & Proteus sp. \\
\hline
\end{tabular}

Number in the parenthesis indicate the reference; MHRC, Medicare Hospital and Research Center. 
infection at the point of time studied. Citrobacter sp. ranked $6^{\text {th }}$ and $7^{\text {th }}$ frequently isolated pathogen in wound infection ${ }^{2,14}$ while none was isolated among 200 specimens processed for bacteriological culture9. Among 197 isolates studied, 2 isolates of Citrbacter sp. were isolated and none of them were MDR. Most of the isolates of Citrobacter sp. isolated in this study were resistant to $\beta$-lactmam antibiotics and were susceptible to sulphoperazone-sulbactam, fluroquinolones and amino glycosides. However Citroabcter sp. susceptible to sulphoperazone-sulbactam combination were also seen in patients presented with pulmonary disease ${ }^{15}$. Among 23 isolates of Citrobacter sp., 20 isolates (86.95) showed MDR phenotype. This study is the first study to highlights the emergence of MDR Citrobacter sp. in the hospital in Kathmandu as major etiology of surgical wound infection. E. coli was the second most common pathogen recovered from surgical wound infection and other pathogens like, S. aureus, Acinetobacter sp., K. pneumoniae were also common.

Although, Acinetobacter sp. and P. aeruginosa, were less frequently isolated in wound infection these are notorious pathogens to cause myriad of nosocomial infections. Carbapenem, colistin, tigecyline and rifampin in combination with these antibiotics are used to treat the multi-drug, pan-drug resistant strains of these pathogens however the resistant strains to all of these antibiotics have already emerged ${ }^{12,16,17}$. Most strains of these pathogens were resistant to $\beta$-lactam antibiotics, amino glycosides, and fluroquinolones but were still susceptible to imipenem. However, one isolates of $\mathrm{E}$. coli was resistant to imipenem. Imipenem although not widely used in clinical practice in Nepal, overuse of this antibiotic may select imipenem resistant strains and these strains could increase and spread.

Nosocomial pathogens are mostly normal flora of the skin, present in the hospital environment (water, air cooler), carried on innate objects (stethoscope, IV drips, IV stands, bed linens) and hands of medical staff ${ }^{15}$. The breech in the skin due to any interventions (surgery, intravenous line, catheters etc) or inappropriate patient care might help the pathogen to lodge in patient's body and gain access to the site of infection. The pre-existing illness like, metabolic diseases, immunosupression, malignancy, and malnutrition have been described as predisposing factors for surgical wound infection. An emergency procedure, length of pre-operative hospitalization, antibiotic prophylaxis, duration of operation, wound class, and wound contamination also significantly contributes to the wound infection ${ }^{18}$.

\section{Conclusion}

This report depicts the reverse trends of pathogen isolation and increasing antibiotic resistance in nosocomial Citrobacter sp. isolated in surgical wound infection. This pathogen is ubiquitous in nature (food, soil, water) and a colonizer of human gastrointestinal tract and can cause difficult-to-treat nosocomial wound and other infections if infection control strategies like, disinfection of wards, contact precaution against infectious versus colonized patients and judicious use of antibiotics are practiced.

\section{References}

1. Haley RW, Schaberg DR, Crossley KB, Von Allmen SD, McGowan JE Jr. Extra charges and prolongation of stay attributable to nosocomial infections: a prospective interhospital comparison. Am J Med 1981;70:51-8.

2. Banjara MR, Sharma AP, Hoshi AB, Yuladhar NR, Ghimire $P$, Bhatta DR. Surgical wound infections in patients of Tribhuvan University Teaching Hospital. Nepal Health Res Counc 2003; 3: 41-5.

3. Pandit A, Sharma P, Yangzom K. Incidence of caesarean wound infection in Patan Hospital, Nepal. J Nepal Med Assoc 2003; 42: 280-3.

4. Drug and therapeutic letter 1999;3: 9-12.

5. B Shrestha, B Pokhrel, T Mohapatra. Study of nosocomial isolates of Staphylococcus aureus with special reference to methicillin resistant $S$. aureus in a tertiary care hospital in Nepal. Nepal Med Coll J 2009; 11: 123-6.

6. Mustafa A, Bukhari A, Kakru DK, Tabish SA, Quadri GJ. Incidence of nosocomial wound infection in post operative patients at teaching hospital in Kashmir.JK- Practitioner 2004; 11: 38-40.

7. Sisirak M, Zvizdic, Hukic M. Methicillin-Resistant Staphylococcus aureus (MRSA) as a cause of nosocomial wound infection. Bos J Basic Med Sci 2010;1: 32-7.

8. Stosor V, Peterson LR, Postelnick M et al. Enterococcus faecium bacteremia: does vancomycin resistance make a difference? Arch Intern Med. 1998; 158:522-7.

9. Bhatta CP, Lakhey M. The distribution of pathogens causing wound infection and their antibiotic susceptibility pattern. J Nepal Health Res Counc 2007;5: 22-6.

10. Coque TM, Novais A, Carattoli A et al. Dissemination of clonally related Escherichia coli strains expressing extended-spectrum $\beta$-lactamase CTX-M-15. Emerging Infect Dis 2008;14:195-200.

11. Kumarasamy K, Toleman MA et al. Emergence of new antibiotic resistance mechanism in India, Pakistan, and the UK: molecular, biological, and epidemiologica study. The lancet 2010 
12. Thapa B, Tribuddharat C, Srifuengfung S, Dhiraputra C. High prevalence of bla ${ }_{\text {OXA-23 }}$ in oligoclonal carbapenem resistant Acinetobacter baumannii from Siriraj Hospital, Madhidol University, Bangkok, Thailand. South East Asian J Trop Med 2010; 41: 625-35.

13. Monica C. District laboratory practices in tropical countries. Part 2. Cambridge University Press.

14. Kansakar P, Pokherel BM, Tuladhar NR. A study on bacteriology of wound infection and the antibiotic sensitivity pattern of the isolates. Fourth congress of association of clinical pathologists of Nepal (ACPN) February 21-2, Souvenir 2003; 35.

15. Thapa B, Adhikari P, Mahat K,Chhetri MR, Joshi LN. Multidrug-resistant nosocomial Citrobacter in a Hospital in Kathmandu. Nepal Med Coll J 2009; 11: 195-9

16. Thapa B, Tribuddharat C, Rugdeekha S, Techachaiwiwat W, Srifuengfung S, Dhiraputra C. Rifampin resistance in carbapenem-resistant Acinetobacter baumannii in Siriraj Hospital, Thailand. Nepal Med Coll J 2009; 11: 232-7.

17. Tribuddharat $C$, Fennewald M. Integron mediated rifampin resistance in Pseudomonas aeruginosa. Antimicrob Agents Chemother 1999; 43: 960-2.

18. PL Nandi, S Soundara Rajan, KC Mak, SC Chan, YP So. Surgical wound infection. HKMJ 1999;5:82-6. 